\title{
Testing variances in wavelet regression models
}

\author{
Alwell J. Oyet*, Brajendra Sutradhar \\ Department of Mathematics and Statistics, Memorial University of Newfoundland, St. John's, \\ Nfld., Canada, AlC $5 S 7$
}

Received October 2001; received in revised form September 2002

\begin{abstract}
In this paper we develop an asymptotically locally optimal partial score test for testing the suitability of a homoscedastic wavelet model against a general heteroscedastic wavelet model. As the construction of the partial score test requires a consistent estimate for the nuisance parameter, namely the constant variance estimate under the null hypothesis, we conduct a comprehensive investigation in order to choose its best possible estimate among some competitors. The size and power performances of the partial score test are reported for testing for heteroscedasticity in a time series of finite length.
\end{abstract}

(c) 2003 Elsevier Science B.V. All rights reserved.

Keywords: Daubechies wavelet; Gasser-Müller estimator; Haar wavelet; Partial score test; Weighted least squares

\section{Introduction}

Wavelet methods have been most widely studied in the nonparametric regression problem of estimating a function $f$ on the basis of observations $y_{i}$ at time points $t_{i}$, modeled as

$$
y_{i}=f\left(t_{i}\right)+\varepsilon_{i} ; \quad i=1, \ldots, n,
$$

where $\varepsilon_{1}, \ldots, \varepsilon_{i}, \ldots, \varepsilon_{n}$ are noise. Traditionally, it is assumed that $\varepsilon_{i} \stackrel{\text { iid }}{\sim} N\left(0, \sigma^{2}\right)$. See for example, Donoho and Johnstone (1995) and Abramovich and Silverman (1998). Some authors, such as Hart and Wehrly (1986), Antoniadis et al. (1994), and Kovac and Silverman (2000) do not assume any distribution for the error terms, rather they assume that $\varepsilon_{i} \stackrel{\text { iid }}{\sim}\left(0, \sigma^{2}\right)$.

Recently, Cai et al. (1998) and Kovac and Silverman (2000, Section 7.1, pp. 181-182) argue that there are situations where the noise may be heteroscedastic. By assuming that $\varepsilon_{i} \sim\left(0, \sigma_{i}^{2}\right)$, for each $i=1, \ldots, n$, Kovac and Silverman (2000) estimated the standard deviation of the $i$ th data

\footnotetext{
${ }^{*}$ Corresponding author. Tel.: +1-709-737-8075; fax: +1-709-737-3010.

E-mail address: aoyet@math.mun.ca (A.J. Oyet).
} 
point by taking the median of the absolute values of $d_{i}=\left(y_{i+1}-y_{i}\right) / \sqrt{2}$ for $i=1, \ldots, n-1$, over a small window of width 0.2 around each point. Note however that the performance of this type of local variance estimates is not adequately addressed in the literature. Moreover, it is clear that if the variances are equal and one attempts to use the estimated $\sigma_{i}^{2}$ to obtain improved estimates of $f$ (see also Silverman, 1985), there will be a substantial loss in efficiency in the estimation of the regression function. This raises the issue of testing the equality of $\sigma_{i}^{2}$ before estimating these parameters. With this in view, Cai et al. (1998) developed a partial score test for testing $\sigma_{i}^{2}=\sigma^{2}$, for all $i=1, \ldots, n$, which requires a consistent estimate of $\sigma^{2}$. In Section 2, we show that there are problems associated with the formulation of the null hypothesis given in Cai et al. (1998). For example, it is clear from their simulation study that the estimate of the constant variance $\sigma^{2}$ proposed by Donoho and Johnstone (1995) may not be consistent, in general, and perform very poorly (see Tables 1 and 3). Müller (1985) suggested to estimate the constant variance $\sigma^{2}$ by fitting constants to successive triples of the data, which was used later on by Antoniadis et al. (1994), among others.

As opposed to the formulation of the null hypothesis used in Cai et al. (1998), in this paper, we first formulate the null hypothesis under the assumption that the local variances used in Kovac and Silverman (2000, pp. 181-182) will be the same in a given time interval (i.e. for a group of observations), and then develop a partial score test for testing the equality of these group variances under normality. Details are provided in Section 2. Note that we also discuss this partial score test for testing the group variances under $t$-distribution for the noises. As our approach also requires the consistent estimation of a constant variance parameter, we consider a number of new estimators for this constant variance in Section 3 and compare their performances along with some existing estimators such as Müller's estimator. This we do in order to choose a best possible constant variance estimator for the construction of the partial score test that we discuss in Section 2. In Section 4, we examine the size and power performances of the proposed test through a simulation study.

\section{Test for homoscedastic versus heteroscedastic wavelet models}

As opposed to the homoscedastic wavelet model (1.1) we now write a general heteroscedastic model given by

$$
y_{i}=f\left(t_{i}\right)+\sigma_{i} \xi_{i} ; \quad i=1, \ldots, n,
$$

where $\xi_{i} \sim N(0,1)$ and $\operatorname{var}\left(\varepsilon_{i}\right)=\operatorname{var}\left(\sigma_{i} \xi_{i}\right)=\sigma_{i}^{2}$. Cai et al. (1998) considered a special type of heteroscedastic model, where it is assumed that the error variances depend on observed covariates through a parametric relationship of known form, such as $\sigma_{i}^{2}=g\left(\mathbf{z}_{i}, \boldsymbol{\delta}\right)$ with $\mathbf{z}_{i}$ as a $p \times 1$ vector of covariates and $\boldsymbol{\delta}$ is a $p \times 1$ vector of parameters, $p$ being small in practice. More specifically, for testing the homoscedasticity, these authors have tested $H_{0}: \boldsymbol{\delta}=\boldsymbol{\delta}_{0}$ so that $g\left(\mathbf{z}_{i}, \boldsymbol{\delta}_{0}\right)=1$ or a constant, say $\sigma_{0}^{2}$. In their simulation study, they considered $g\left(\mathbf{z}_{i}, \boldsymbol{\delta}\right)=\exp \left(\mathbf{z}_{i} \boldsymbol{\delta}\right)$ and tested for homoscedasticity by testing the null hypothesis $H_{0}: \boldsymbol{\delta}=\boldsymbol{\delta}_{0}=\mathbf{0}$, a zero vector, so that $g\left(\mathbf{z}_{i}, \boldsymbol{\delta}_{0}=\mathbf{0}\right)=1$ for $i=1, \ldots, n$. Note that this formulation of the null hypothesis appear to have serious limitations. First, if it is considered that the noise variances depend on the covariates, it is then reasonable to assume that for $\mathbf{z}_{i}=\mathbf{z}$, say, for all $i=1, \ldots, n$ one would expect that $\sigma_{i}^{2}=g\left(\mathbf{z}_{i}, \boldsymbol{\delta}\right)$ will be the same. Now, for $\mathbf{z}_{i}=\mathbf{z}$, it is still possible to have $g\left(\mathbf{z}_{i}, \boldsymbol{\delta}_{0}=\mathbf{0}\right)=g\left(\mathbf{z}, \boldsymbol{\delta}_{0} \neq \mathbf{0}\right)=1$. Thus, this formulation appears to suffer from 
identification problems since we can find other non-zero $\delta$ which can guarantee homoscedasticity. Moreover, it is quite restrictive to assume that the variance function ' $g$ ' is known.

In this paper, we follow the idea of local variance described by Kovac and Silverman (2000, pp. 181-182) but unlike these authors, we assume that a group of observations have the same local variance. Suppose that there are $q$ such local groups with variances $\sigma_{(1)}^{2}, \ldots, \sigma_{(j)}^{2}, \ldots, \sigma_{(q)}^{2}$. Note that from testing point of view, $q$ ranges from 2 to $n$. Kovac and Silverman (2000), for example, assumed that the local variances may be different at every time point. Thus in their case, $q=n$. One of the main difficulties of this general consideration is that these variances may not be estimated consistently. Moreover, in a time series process, it is likely that a number of neighbouring observations will have constant variance. For example, in a daily temperature series recorded in a year, it may be sensible to assume that variability in the temperature readings in a specific season is constant but the variation will be different as the season changes. Thus, it would be reasonable to consider $q=4$ groups based on four seasons although here $n=365$. In more technical problems such as in signal processing, one may observe several interventions or an unusual peak or trough after an interval of smooth signals. Consequently, if there are $q-1$ such possible interventions, one could choose to test the variabilities in $q$ groups where $2 \leqslant q<n$. Note however that although $n$ is usually large, a selection of small groups, in general, ensures the consistency of the variance estimators for these groups. In summary, the motivation to choose a particular value for $q$ is heuristic and the choice will largely depend on the nature of the data as well as on previous experiences of the experimenter with such data.

Let $n_{j}(j=1,2, \ldots, q)$ be the number of observations in the $j$ th local group so that $\sum_{j=1}^{q} n_{j}=n$, where $n$ is the length of the time series considered under the model (1.1) or (2.1). In terms of these new variances, the covariance matrix of $\boldsymbol{\varepsilon}=\left(\varepsilon_{1}, \ldots, \varepsilon_{i}, \ldots, \varepsilon_{n}\right)^{\prime}=\left(\sigma_{1} \xi_{1}, \ldots, \sigma_{i} \xi_{i}, \ldots, \sigma_{n} \xi_{n}\right)^{\prime}$, say $\boldsymbol{\Sigma}$, has the form given by

$$
\boldsymbol{\Sigma}=\operatorname{diag}\left[\sigma_{1}^{2}, \ldots, \sigma_{i}^{2}, \ldots, \sigma_{n}^{2}\right]=\operatorname{diag}\left[\sigma_{(1)}^{2} \mathbf{1}_{n_{1}}^{\prime}, \ldots, \sigma_{(j)}^{2} \mathbf{1}_{n_{j}}^{\prime}, \ldots, \sigma_{(q)}^{2} \mathbf{1}_{n_{q}}^{\prime}\right],
$$

where $\mathbf{1}_{n_{j}}$ is the $n_{j}$-dimensional unit vector. Now, to test for homoscedasticity in the wavelet model (2.1) one may write the null hypothesis $H_{0}: \sigma_{(1)}^{2}=\cdots=\sigma_{(j)}^{2}=\cdots=\sigma_{(q)}^{2}$, which, through the reparametrization $\gamma_{j}=\sigma_{(j)}^{2} / \sigma_{(q)}^{2}$, reduces to

$$
H_{0}: \gamma_{1}=\cdots=\gamma_{j}=\cdots=\gamma_{q-1}=1 \text {. }
$$

We note that in the context of time series models, the $f\left(t_{i}\right)$ in $(2.1)$ represent deterministic trend or seasonal effects which may be a polynomial or a trigonometric function (see Abraham and Ledolter, 1983, Chapters 2-4). In addition to this consideration, when it is assumed that $\varepsilon_{i}$ are heterogeneous but uncorrelated, the $y_{i}$ series may be treated as arising from a deterministic time series model. The present testing approach is however flexible and can accomodate correlated errors such as autoregressive moving average (ARMA) and/or seasonal autoregressive moving average (SARMA) errors with nonconstant variances (see Sutradhar and Dagum, 1998). But, any such additional assumption will require the estimation of the nuisance correlation parameters which is beyond the scope of this paper.

In the following section, we develop a partial score test for testing the null hypothesis $H_{0}$ in (2.3). Note that the partial score test we develop here goes back to Neyman (1959), which was used by many authors in the econometrics literature such as Breusch and Pagan (1979) and Koenker (1981). Thus, Cai et al. (1998) in fact have used the Neyman's partial score test. The Neyman's (1959) score test is asymptotically unbiased in estimating a preassigned level of significance. Also, this test 
is asymptotically locally most powerful and in general asymptotically equivalent to the likelihood ratio and Wald's tests (Moran, 1970). In the present context, we prefer Neyman's score test as this requires only a consistent estimate for the nuisance parameter $\sigma_{(q)}^{2}$, which need not be a maximum likelihood estimate.

\subsection{Partial score test for homoscedasticity}

To test for homoscedasticity, we test $H_{0}$ in (2.3). Since $\sigma_{(q)}^{2}$ in $\gamma_{j}=\sigma_{(j)}^{2} / \sigma_{(q)}^{2}$ is unknown in practice, it then becomes a nuisance parameter, whereas $\gamma_{j}$ for $j=1, \ldots, q-1$ are the main parameters of interest for the test. Note that by using $\sigma_{(j)}^{2}=\sigma_{(q)}^{2} \gamma_{j}$ in (2.2), we obtain

$$
\boldsymbol{\Sigma}=\sigma_{(q)}^{2} \operatorname{diag}\left[\gamma_{1} \mathbf{1}_{n_{1}}^{\prime}, \ldots, \gamma_{j} \mathbf{1}_{n_{j}}^{\prime}, \ldots, \mathbf{1}_{n_{q}}^{\prime}\right]
$$

which under $H_{0}$ in (2.3), reduces to $\boldsymbol{\Sigma}=\sigma_{(q)}^{2} \mathbf{I}_{n}$ where $\mathbf{I}_{n}$ is the $n \times n$ identity matrix.

In terms of new groupings, let $t_{i}$ in (1.1) or (2.1) represent the $h$ th $\left(h=1, \ldots, n_{j}\right)$ time points of the $j$ th $(j=1, \ldots, q)$ group such that $t_{j h} \equiv t_{\left\{(j-1) \sum_{u=1}^{j} n_{u}+h\right\}} \equiv t_{i}$ for all $h=1, \ldots, n_{j}$ under the $j$ th $(j=1, \ldots, q)$ group. It then follows from (2.1) and (2.4) that the log likelihood of the parameters $\gamma_{j}(j=1, \ldots, q-1)$ and $\sigma_{(q)}^{2}$, under the normal distribution may be expressed as

$$
\mathscr{L}=-\frac{1}{2}\left[n \log (2 \pi)+n \log \sigma_{(q)}^{2}+\sum_{j=1}^{q} n_{j} \log \gamma_{j}+\left\{\sigma_{(q)}^{2}\right\}^{-1} \sum_{j=1}^{q} \sum_{h=1}^{n_{j}} \gamma_{j}^{-1}\left(y_{j h}-f\left(t_{j h}\right)\right)^{2}\right],
$$

where $\gamma_{q}=1$ and $y_{j h}$ and $f\left(t_{j h}\right)$ are, respectively, the observations and the value of the non-parametric function at time point $t_{j h}$. Define the vector $\gamma=\left(\gamma_{1}, \ldots, \gamma_{q-1}\right)^{\prime}$ and let $\hat{\sigma}_{(q)}^{2}$ be a consistent estimator of $\sigma_{(q)}^{2}$. Neyman's partial score test is based on

$$
U_{j}\left(\hat{\sigma}_{(q)}^{2}\right)=\eta_{j}\left(\hat{\sigma}_{(q)}^{2}\right)-\psi_{j 1} \xi_{1}\left(\hat{\sigma}_{(q)}^{2}\right), \quad j=1, \ldots, q-1,
$$

where $\psi_{j 1}$ is the partial regression coefficient of $\eta_{j}$ on $\xi_{1}$, and where for $j=1, \ldots, q-1, \eta_{j}$ and $\xi_{1}$ may be simplified to obtain

$$
\eta_{j}=\left.\frac{\partial \mathscr{L}}{\partial \gamma_{j}}\right|_{\gamma=\mathbf{1}_{q-1}}=\frac{1}{2}\left[\frac{\sum_{h=1}^{n_{j}}\left(y_{j h}-f\left(t_{j h}\right)\right)^{2}}{\sigma_{(q)}^{2}}-n_{j}\right],
$$

and

$$
\xi_{1}=\left.\frac{\partial \mathscr{L}}{\partial \sigma_{(q)}^{2}}\right|_{\gamma=\mathbf{1}_{q-1}}=\frac{1}{2 \sigma_{(q)}^{2}}\left[\frac{\sum_{j=1}^{q} \sum_{h=1}^{n_{j}}\left(y_{j h}-f\left(t_{j h}\right)\right)^{2}}{\sigma_{(q)}^{2}}-n\right],
$$

respectively. After the evaluation of $\psi_{j 1}$, the score function in (2.5) may be used to construct the score vector

$$
\mathbf{u}=\left[u_{1}\left(\sigma_{(q)}^{2}\right), \ldots, u_{q-1}\left(\sigma_{(q)}^{2}\right)\right]^{\prime}=\boldsymbol{\eta}-\mathbf{d} g^{-1} \xi_{1},
$$


where $\boldsymbol{\eta}=\left(\eta_{1}, \ldots, \eta_{j}, \ldots, \eta_{q-1}\right)^{\prime}$ with $\eta_{j}$ as given in (2.6). In $(2.8), \mathbf{d}=\left(d_{1}, \ldots, d_{j}, \ldots, d_{q-1}\right)^{\prime}$ is a $(q-$ 1)-dimensional vector with its $j$ th $(j=1, \ldots, q-1)$ element defined as $d_{j}=-\left.E\left\{\partial^{2} \mathscr{L} / \partial \gamma_{j} \partial \sigma_{(q)}^{2}\right\}\right|_{\gamma=\mathbf{1}_{q-1}}=$ $\left(2 \sigma_{(q)}^{2}\right)^{-1} n_{j}$ and $g$ is a scalar quantity given by $g=-\left.E\left\{\partial^{2} \mathscr{L} / \partial \sigma_{(q)}^{4}\right\}\right|_{\gamma=\mathbf{1}_{q-1}}=\left(2 \sigma_{(q)}^{4}\right)^{-1} n$. Next, it can be shown that the covariance matrix of $\mathbf{u}$ in (2.8) is given by $\mathbf{C}-\mathbf{d} g^{-1} \mathbf{d}^{\prime}$, where $\mathbf{C}$ is a diagonal matrix with elements $c_{j k}=-\left.E\left\{\partial^{2} \mathscr{L} / \partial \gamma_{j} \partial \gamma_{k}\right\}\right|_{\gamma=\mathbf{1}_{q-1}}=n_{j} / 2$, for $j=k$. After replacing $\sigma_{(q)}^{2}$ in $\mathbf{u}, \mathbf{C}, \mathbf{d}$ and $g$ by $\hat{\sigma}_{(q)}^{2}$, one then obtains Neyman's partial score test statistic as

$$
s\left(\hat{\sigma}_{(q)}^{2}\right)=\mathbf{u}^{\prime}\left[\mathbf{C}-\mathbf{d} g^{-1} \mathbf{d}^{\prime}\right]^{-1} \mathbf{u},
$$

which is asymptotically distributed as $\chi^{2}$ with $q-1$ degrees of freedom.

Now to examine the performance of this test statistic (2.9) under a different distribution than normal, we choose a heavy-tailed $t$-distribution with the p.d.f of $y_{i}$ given by

$$
p\left(y_{i}\right)=\frac{(v-2)^{v / 2} \Gamma\{(v+1) / 2\}}{\sqrt{\pi} \Gamma(v / 2)}\left(\sigma_{i}^{2}\right)^{-1 / 2}\left[(v-2)+\left\{\left(y_{i}-f\left(t_{i}\right)\right) / \sigma_{i}\right\}^{2}\right]^{-(v+1) / 2}
$$

for a given degrees of freedom $v>2$. Using the same notations as before, it is straightforward to verify that apart from a constant, $\eta_{j}$ and $\xi_{1}$ are linear functions of independent quantities under both distributions. Note that the construction of the test statistic (2.9), in the $t$-case, is immediate once $\eta_{j}$ and $\xi_{1}$ are computed. These linear functions in the $t$-case consist of complicated ratio variables as opposed to the normal case. Thus, for certain non-smooth nonparametric functions, the normal approximation in the $t$-case might require much larger samples as compared to the normal case. This is evident from our simulation which will be reported later.

In the next section, we examine the performance of several approaches in estimating the common wavelet variance $\sigma_{(q)}^{2}$, through a simulation study. The best variance estimate is then used in Section 4 to examine the size and power performances of the score statistic $s\left(\hat{\sigma}_{(q)}^{2}\right)$ in testing the homoscedasticity of the wavelet variances.

\section{Null consistent estimator for the wavelet variance}

Recall that if the null hypothesis is true then the variances of all the local groups become identical, i.e., $\sigma_{(j)}^{2}=\sigma_{(q)}^{2}=\sigma^{2}$, say, for $j=1, \ldots, q-1$. The construction of the test statistic (2.9) requires the consistent estimation of this nuisance parameter $\sigma_{(q)}^{2}=\sigma^{2}$. Müller (1985) proposed to use the estimate

$$
\hat{\sigma}_{\mathrm{M}}^{2}=\frac{2}{3(n-2)} \sum_{i=2}^{n-1}\left[Y_{i}-\frac{1}{2}\left(Y_{i-1}+Y_{i+1}\right)\right]^{2},
$$

obtained by fitting constants to successive triples of the data. This estimator (3.1) was later used by Antoniadis et al. (1994) to construct a confidence interval for the regression function $f(t)$. Donoho and Johnstone (1995, p. 1218) proposed to use the robust estimator

$$
\hat{\sigma}_{\mathrm{DJ}}=\frac{\operatorname{median}\left(\left|\tilde{\omega}_{J-1, k}\right|: 0 \leqslant k<2^{J-1}\right)}{0.6745},
$$


for the estimation of $\sigma$, where $\left(\tilde{\omega}_{j, k}\right)$ are the wavelet coefficients of $y_{i}\left(i=0,1, \ldots, 2^{J}-1\right)$, indexed dyadically through the scheme $\tilde{\omega}_{j, k}: j=0, \ldots, J-1 ; k=0, \ldots, 2^{j}-1$. This estimator has also been used recently by Cai et al. (1998) in connection with the score tests for heteroscedasticity in wavelet regression. We remark that the estimators (3.1) and (3.2) are consistent for $\sigma^{2}$ under certain restrictions, but nothing is known about the efficiency of these estimators. With a view to obtaining an improved variance estimator, we now introduce three estimators which are weighted averages of the estimated squared residuals, the weights being the same as those of the weights we use in the estimation of the wavelet regression function $f(t)$. The estimators are defined as

$$
\begin{aligned}
& \hat{\sigma}_{\mathrm{MGM}, 1}^{2}=\frac{\sum_{i=1}^{n} \sum_{k=1}^{n} w_{\mathrm{MGM}, i}\left(t_{k}\right) e^{2}\left(t_{k}\right)}{\sum_{i=1}^{n} \sum_{k=1}^{n} w_{\mathrm{MGM}, i}\left(t_{k}\right)}, \\
& \hat{\sigma}_{\mathrm{MGM}, 2}^{2}=\frac{\sum_{k=1}^{n} w_{\mathrm{MGM}, k}\left(t_{k}\right) e^{2}\left(t_{k}\right)}{\sum_{k=1}^{n} w_{\mathrm{MGM}, k}\left(t_{k}\right)}, \\
& \hat{\sigma}_{\mathrm{WLS}}^{2}=\frac{\sum_{k=1}^{n} w_{\mathrm{WLS}, k}\left(t_{k}\right) e^{2}\left(t_{k}\right)}{\sum_{k=1}^{n} w_{\mathrm{WLS}, k}\left(t_{k}\right)},
\end{aligned}
$$

where $\hat{\sigma}_{\mathrm{MGM}, 1}^{2}$ and $\hat{\sigma}_{\mathrm{MGM}, 2}^{2}$ will be referred to as the modified Gasser-Müller estimator of type 1 and 2 respectively, whereas $\hat{\sigma}_{\mathrm{WLS}}^{2}$ will be referred to as the weighted least squares estimator of $\sigma^{2}$. In (3.3)-(3.5), e $\left(t_{k}\right)=f\left(t_{k}\right)-\hat{f}\left(t_{k}\right)$ are the residuals for $k=1, \ldots, n$, where $\hat{f}\left(t_{k}\right)$ is a suitable estimator of $f\left(t_{k}\right)$, which may be expressed as

$$
\hat{f}\left(t_{k}\right)=\sum_{i=1}^{n} w_{i}\left(t_{k}\right) y_{k},
$$

where $w_{i}\left(t_{k}\right)$ are weights constructed at time point $t_{k}$, for $i=1, \ldots, n$. Consequently, if $f\left(t_{k}\right)$ is estimated, for example, by using a wavelet version of the well-known Gasser-Müller wavelet estimator, then $w_{i}\left(t_{k}\right)$ will be denoted by $w_{\mathrm{GM}, i}\left(t_{k}\right)$. It is then clear that to obtain $w_{\mathrm{MGM}, i}\left(t_{k}\right)$ for $(3.3)$ and (3.4), we will estimate the regression function $f\left(t_{k}\right)$ by using a modified wavelet version of the Gasser-Müller estimator. Similarly, to obtain the weighted least squares weights $w_{\mathrm{WLS}, i}\left(t_{k}\right)$ for (3.5) we require to estimate $f\left(t_{k}\right)$ by using a suitable weighted least squares wavelet approach. The constructions of these two types of weights are discussed briefly below.

\subsection{Construction of weights}

To construct the weights of the wavelet version of the Gasser-Müller and weighted least squares estimators, we first express $f(t)$ in terms of its finite order $m$ wavelet expansion and rewrite the model (1.1) as

$$
y_{i}=\mathbf{q}_{m}^{\mathrm{T}}\left(t_{i}\right) \boldsymbol{\beta}+g\left(t_{i}\right)+\varepsilon_{i}, \quad i=1, \ldots, n,
$$


for $t_{i} \in\left[A_{i-1}, A_{i}\right]$ so that $\bigcup_{i=1}^{n}\left[A_{i-1}, A_{i}\right]=[0,1]$. In $(3.7), q_{m}(t)$ is a $2^{m+1} \times 1$ vector consisting of a system of dilated and translated versions $\psi^{-l, k}(t)=2^{l / 2} \psi\left(2^{l} t-k\right),\left(l=0, \ldots, m ; k=0, \ldots, 2^{l}-1\right)$ of a primary wavelet $\psi(t)$ and a scaling function $\phi(t)$. For instance, the Haar scaling function is defined by $\phi(t)=I_{[0,1)}(t)$ and the primary wavelet is $\psi(t)=\phi(2 t)-\phi(2 t-1)$, where $I(t)$ is the indicator function. The term $g(t)$ represents the wavelet terms not used in the representation of $f(t)$. Thus, in actual computations only the first term in (3.7) can be estimated. We note that in practice, the choice of a value for $m$ and the wavelet to be used will depend on the complexity and on the structure of $f(t)$. For example, the Haar wavelet system is known to be most suitable for describing block or step functions because of the block pattern in the Haar primary wavelet and the Daubechies wavelet has been found to be very useful in estimating smooth functions. More details on the choice of $m$ and wavelets and its applications in statistics can be found in Antoniadis et al. (1994) and Härdle et al. (1998, pp. 125-191).

Now, in terms of the notations in (3.7), weights for the Gasser-Müller estimator may be defined as $w_{\mathrm{GM}, i}(t)=\int_{A_{i-1}}^{A_{i}} \mathbf{q}_{m}^{\mathrm{T}}(t) \mathbf{q}_{m}(s) \mathrm{d} s, i=1, \ldots, n$, at a time point $t$. These weights can further be modified to obtain better estimates $\hat{f}(t)$ (3.6), where the modified weights are

$$
w_{\mathrm{MGM}, i}(t)=\int_{A_{i-1}}^{A_{i}} \mathbf{q}_{m}^{\mathrm{T}}(t) \mathbf{B}^{-1} \mathbf{q}_{m}(s) \mathrm{d} s,
$$

with $\mathbf{B}=\sum_{i=1}^{n} \int_{A_{i-1}}^{A_{i}} \mathbf{q}_{m}(s) \mathbf{q}_{m}^{\mathrm{T}}\left(t_{i}\right) \mathrm{d} s$. For the construction of the weights used in weighted least squares estimation of $f(t)$, we follow Oyet and Wiens (2000) and write the weights as

$$
w_{\mathrm{WLS}, i}(t)=\frac{\int_{0}^{1}\left\|\mathbf{q}_{m}(s)\right\| \mathrm{d} s}{\left\|\mathbf{q}_{m}\left(t_{i}\right)\right\|},
$$

where $\|\mathbf{q}(s)\|$ denotes the Euclidean norm. As indicated earlier, these two types of weights given in (3.8) and (3.9) may be used in (3.3)-(3.5) to obtain improved estimators for $\sigma^{2}$.

In order to have some feelings about the performance of these weights (3.8) and (3.9), we have used them in (3.6) to estimate, for example, three functions as shown in Fig. 1. The Haar and Daubechies wavelet systems were used for estimation. It appears from Fig. 1 that both the modified Gasser-Müller and the weighted least squares weights work well in estimating the selected functions. In the following sub-section, we examine the performance of the variance estimators (3.1)-(3.5) through a simulation study.

\subsection{Choosing variance estimator: a simulation study}

Three different functions were used to investigate and compare the performance of the five variance estimators. These functions include (1) constant function, $f(t)=3 ;(2)$ a balanced block function consisting of 16 means defined by $f(t)=\sum_{j=1}^{16} h_{j} I_{\left[2^{-p}(j-1), 2^{-p} j\right)}(t), p=4, h_{j}=(-1.2,0.5,3,2,4,1.5$, $-1.2,0.5,3,2,4,1.5,3,2,4,1.5)$, where $I_{\left[2^{-p}(j-1), 2^{-p} j\right)}(t)$ is the indicator function and (3) the HeaviSine function defined by $f(t)=4 \sin 4 \pi t-\operatorname{sgn}(t-0.3)-\operatorname{sgn}(0.72-t)$. 


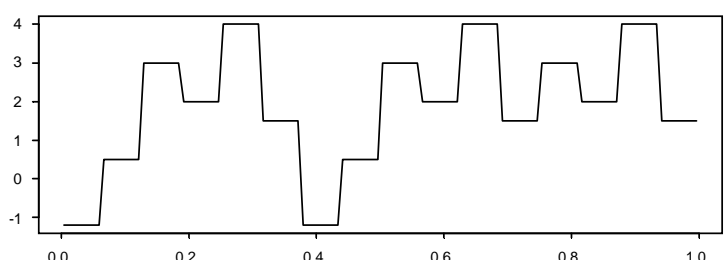

(a)

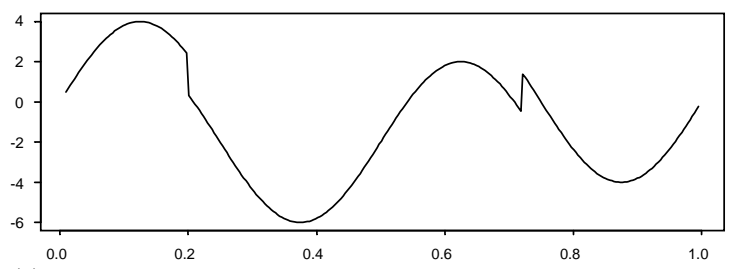

(c)

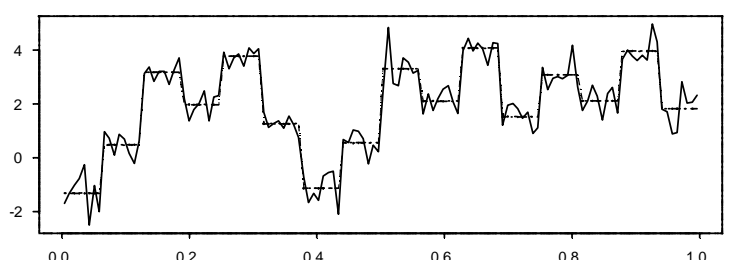

(b)

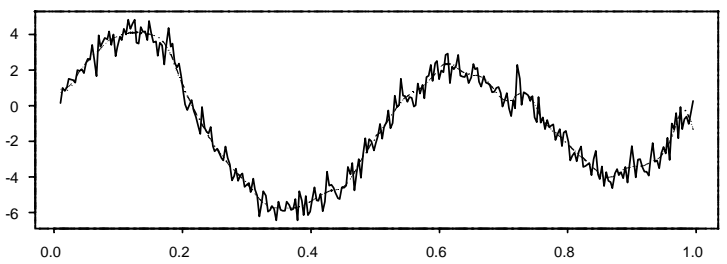

(d)

Fig. 1. Plot of (a) Balanced 16 blocks mean function $f(t)$, (b) Generated data from balanced block function (solid line), modified GM estimate (dotted line) and WLS estimate (broken line), (c) HeaviSine mean function $f(t)$, (d) Generated data from HeaviSine function (solid line), modified GM estimate (dotted line) and WLS estimate (broken line).

A data set of 128 points was generated from the constant and balanced block functions. We then used the Haar wavelet representation with $m=0$ and $m=3$ to estimate these functions, respectively, $m$ being the order of wavelet expansion. For the HeaviSine function, we generated 300 data points and used the Daubechies wavelet representation with $m=4$, in order to estimate the corresponding function. Next, by constructing the required weights, as in Section 3.1 and by using the data, we compute all five variance estimators given by Eqs. (3.1)-(3.5). The whole estimation procedure is repeated 500 times. Table 1 shows the average of each of the five variance estimators computed from these 500 simulations, for selected values of $\sigma^{2}$. The values in parentheses are the standard deviations of the estimators from the 500 simulations.

The results in Table 1 demonstrate that $\hat{\sigma}_{\mathrm{DJ}}^{2}$ (3.2), performs very poorly. Consequently, unlike Cai et al. (1998), we have decided not to use this estimator for the construction of the partial score statistic (2.9) for testing for homoscedasticity. All other four estimators appear to produce unbiased estimators for $\sigma^{2}$ under the constant function model. For other models, the estimators (3.3)-(3.5) appear to perform the same for $\sigma^{2}$ estimation, whereas, $\hat{\sigma}_{\mathrm{M}}^{2}$ (3.1), shows slightly different performance. To be specific, Müller's approach produces unbiased estimates for $\sigma^{2}$ under the HeaviSine model but overestimates $\sigma^{2}$ under the model with balanced blocks, the overestimation problem being serious for small $\sigma^{2}$. The MGM and WLS estimators appear to underestimate $\sigma^{2}$ under the balanced block and HeaviSine models.

In summary, as the underestimation by MGM or WLS approach is negligible for the HeaviSine model and because these approaches in general produce $\sigma^{2}$ estimates with smaller bias than the Müller estimate under the balanced block model, one may choose one of these two estimators to construct the partial score test statistic (2.9). 
Table 1

Simulated average of variance estimator based on 5 different methods for selected values of the constant variance parameter

\begin{tabular}{|c|c|c|c|c|c|}
\hline Function & Method & $\sigma^{2}=0.25$ & $\sigma^{2}=0.64$ & $\sigma^{2}=1$ & $\sigma^{2}=2.25$ \\
\hline \multirow[t]{10}{*}{ Constant } & \multirow{2}{*}{$\hat{\sigma}_{\mathrm{MGM}, 1}^{2}$} & 0.2488 & 0.6281 & 0.9760 & 2.2230 \\
\hline & & $(0.031)$ & $(0.081)$ & $(0.124)$ & $(0.286)$ \\
\hline & \multirow[t]{2}{*}{$\hat{\sigma}_{\mathrm{MGM}, 2}^{2}$} & 0.2488 & 0.6281 & 0.9760 & 2.2220 \\
\hline & & $(0.031)$ & $(0.081)$ & $(0.123)$ & $(0.286)$ \\
\hline & \multirow[t]{2}{*}{$\hat{\sigma}_{\mathrm{WLS}}^{2}$} & 0.2488 & 0.6281 & 0.9759 & 2.2220 \\
\hline & & $(0.031)$ & $(0.081)$ & $(0.124)$ & $(0.286)$ \\
\hline & \multirow[t]{2}{*}{$\hat{\sigma}_{\mathrm{M}}^{2}$} & 0.2547 & 0.6313 & 0.9879 & 2.2566 \\
\hline & & $(0.044)$ & $(0.119)$ & $(0.179)$ & $(0.404)$ \\
\hline & \multirow[t]{2}{*}{$\hat{\sigma}_{\mathrm{DJ}}^{2}$} & 0.0046 & 0.0125 & 0.0168 & 0.0147 \\
\hline & & $(0.006)$ & $(0.016)$ & $(0.024)$ & $(0.057)$ \\
\hline \multirow[t]{10}{*}{ Balanced block } & \multirow[t]{2}{*}{$\hat{\sigma}_{\mathrm{MGM}, 1}^{2}$} & 0.2205 & 0.5613 & 0.8733 & 1.9924 \\
\hline & & $(0.029)$ & $(0.079)$ & $(0.111)$ & $(0.261)$ \\
\hline & \multirow{2}{*}{$\hat{\sigma}_{\mathrm{MGM}, 2}^{2}$} & 0.2205 & 0.5611 & 0.8728 & 1.9926 \\
\hline & & $(0.029)$ & $(0.079)$ & $(0.111)$ & $(0.262)$ \\
\hline & \multirow[t]{2}{*}{$\hat{\sigma}_{\mathrm{WLS}}^{2}$} & 0.2204 & 0.5611 & 0.8729 & 1.9915 \\
\hline & & $(0.028)$ & $(0.079)$ & $(0.111)$ & $(0.261)$ \\
\hline & \multirow[t]{2}{*}{$\hat{\sigma}_{\mathrm{M}}^{2}$} & 0.4133 & 0.7973 & 1.1548 & 2.4514 \\
\hline & & $(0.062)$ & $(0.142)$ & $(0.196)$ & $(0.434)$ \\
\hline & \multirow{2}{*}{$\hat{\sigma}_{\mathrm{DJ}}^{2}$} & 0.2008 & 0.2072 & 0.2132 & 0.2124 \\
\hline & & $(0.039)$ & $(0.05)$ & $(0.063)$ & $(0.08)$ \\
\hline \multirow[t]{10}{*}{ HeaviSine } & \multirow[t]{2}{*}{$\hat{\sigma}_{\mathrm{MGM}, 1}^{2}$} & 0.2603 & 0.6111 & 0.9311 & 2.0607 \\
\hline & & $(0.028)$ & $(0.059)$ & $(0.087)$ & $(0.191)$ \\
\hline & \multirow[t]{2}{*}{$\hat{\sigma}_{\mathrm{MGM}, 2}^{2}$} & 0.2500 & 0.6035 & 0.9184 & 2.0311 \\
\hline & & $(0.023)$ & $(0.055)$ & $(0.082)$ & $(0.184)$ \\
\hline & \multirow[t]{2}{*}{$\hat{\sigma}_{\mathrm{WLS}}^{2}$} & 0.2592 & 0.6096 & 0.9289 & 2.0554 \\
\hline & & $(0.028)$ & $(0.06)$ & $(0.087)$ & $(0.196)$ \\
\hline & \multirow[t]{2}{*}{$\hat{\sigma}_{\mathrm{M}}^{2}$} & 0.2608 & 0.6511 & 1.011 & 2.2697 \\
\hline & & $(0.029)$ & $(0.077)$ & $(0.118)$ & $(0.258)$ \\
\hline & \multirow[t]{2}{*}{$\hat{\sigma}_{\mathrm{DJ}}^{2}$} & 0.0012 & 0.0028 & 0.0042 & 0.0090 \\
\hline & & $(0.002)$ & $(0.003)$ & $(0.004)$ & $(0.007)$ \\
\hline
\end{tabular}

\section{Size and power performances of the score test}

Recall from Section 2 that based on the idea of the same local variance for a group of observations, we have developed the partial score test (2.9) for testing (2.3). In what follows, we examine the size and power performance of (2.9).

\subsection{Sizes of the score tests}

We considered $q=4$ groups of observations and computed the values of the score statistic $s\left(\hat{\sigma}_{(q)}^{2}\right)$ in 1000 simulations, when $\sigma_{(q)}^{2}=\sigma^{2}$ is estimated by (3.1), and (3.3)-(3.5). We thus considered four 
Table 2

Size estimates $(\%)$ of $s_{\mathrm{MGM}}, s_{\mathrm{WLS}}, s_{\mathrm{M} 1}$ and $s_{\mathrm{M} 2}$ tests of $H_{0}: \gamma_{1}=\gamma_{2}=\gamma_{3}=1$ at nominal $5 \%$ level, with $\sigma_{(4)}^{2}=\sigma^{2}=0.80$

\begin{tabular}{|c|c|c|c|c|c|}
\hline \multirow[t]{2}{*}{ Mean function } & \multirow[t]{2}{*}{$n$} & \multicolumn{4}{|l|}{ Test } \\
\hline & & $s_{\mathrm{MGM}}$ & $s_{\mathrm{WLS}}$ & $s_{\mathrm{M} 1}$ & $s_{\mathrm{M} 2}$ \\
\hline Constant & 128 & 0.043 & 0.043 & 0.055 & 0.056 \\
\hline \multirow[t]{2}{*}{16 balanced blocks } & 128 & 0.081 & 0.081 & 0.009 & 0.009 \\
\hline & 256 & 0.051 & 0.051 & 0.017 & 0.017 \\
\hline HeaviSine & 300 & 0.076 & 0.076 & 0.048 & 0.047 \\
\hline
\end{tabular}

statistics $s_{\mathrm{MGM}}, s_{\mathrm{WLS}}, s_{\mathrm{M} 1}$ and $s_{\mathrm{M} 2}$, where $s_{\mathrm{MGM}}$ and $s_{\mathrm{WLS}}$ represent the test statistic (2.9) with $\sigma^{2}$ estimated by (3.3) or (3.4) and (3.5) respectively. The last two statistics represent the same statistic (2.9) with $\sigma^{2}$ estimated by (3.1) but the residual vector $\mathbf{u}$ in (2.9) is computed by using the wavelet function estimated by MGM and WLS approaches, respectively. Table 2 reports size estimates of the normal distribution based score statistics $s_{\mathrm{MGM}}, s_{\mathrm{WLS}}, s_{\mathrm{M} 1}$ and $s_{\mathrm{M} 2}$, for testing the null hypothesis $H_{0}: \gamma_{1}=\gamma_{2}=\gamma_{3}=1$, with nominal level of $5 \%$ so that $\operatorname{Pr}\left(s\left(\hat{\sigma}^{2}\right)>\chi_{q-1}^{2}\right)=0.05$.

It is clear from Table 2 that both $s_{\mathrm{MGM}}$ and $s_{\mathrm{WLS}}$ generally performed well in controlling the size. The statistics $s_{\mathrm{M} 1}$ and $s_{\mathrm{M} 2}$ also perform well under the models with the constant and HeaviSine function but very conservative under the model with balanced block function. This can be attributed to the inflated Müller's variance estimate $\hat{\sigma}_{\mathrm{M}}^{2}$ under the model with the balanced function as shown in Table 1.

The size of the $t$-distribution based score test for $v=3$ were found to be quite dependent on the nonparametric functional form and the length of the series as well. To be specific, the size of the test for the constant function with $n=128$ and balanced block function with $n=512$ produced sizes in the range of $0.059-0.086$. For balanced block function with $n=256$ the sizes were however much higher than 0.05 , which appear to confirm the need for larger time series under the $t$-distribution. The sizes for the HeavySine function were also much higher than 0.05 . Thus the score statistic under the $t$-distribution was found to be liberal as compared to the normal case.

\subsection{Powers of the tests}

This section presents a simulation study of the test's power performances for testing the null hypothesis $H_{0}: \sigma_{(1)}^{2}=\sigma_{(2)}^{2}=\sigma_{(3)}^{2}=\sigma_{(4)}^{2}$, i.e., $\gamma_{1}=\gamma_{2}=\gamma_{3}=1$ where $\gamma_{j}=\sigma_{(j)}^{2} / \sigma_{(4)}^{2}$ against the alternatives specified by the parameter sets $\Omega_{1}: \gamma_{1}=1, \gamma_{2}=1, \gamma_{3}=2 ; \Omega_{2}: \gamma_{1}=1, \gamma_{2}=3, \gamma_{3}=3$; $\Omega_{3}: \gamma_{1}=4, \gamma_{2}=3, \gamma_{3}=2 ; \Omega_{4}: \gamma_{1}=1, \gamma_{2}=1, \gamma_{3}=4$. The power estimates reported in Table 3 represent, for each parameter setting, the proportion of 1000 simulation runs in which the test statistic exceeded the nominal $5 \%$ point of the $\chi^{2}$ distribution with $q-1=3$ degrees of freedom.

It is clear from the table that all three, MGM, WLS and Müller approaches perform almost the same and their power performances are quite satisfactory. Note that although the Müller tests (M1 and M2) has a satisfactory power, it was shown in Table 2 that these tests may be too conservative in controlling the size, for example for the balanced block function. Furthermore, it is clear from 
Table 3

Power estimates $(\%)$ of $s_{\mathrm{MGM}}, s_{\mathrm{WLS}}, s_{\mathrm{M} 1}$ and $s_{\mathrm{M} 2}$ tests of $H_{0}: \gamma_{1}=\gamma_{2}=\gamma_{3}=1$ versus specified alternatives at nominal $5 \%$ level

\begin{tabular}{|c|c|c|c|c|c|c|}
\hline \multirow[t]{2}{*}{ Alternative set } & \multirow[t]{2}{*}{ Mean function } & \multirow[t]{2}{*}{$n$} & \multicolumn{4}{|l|}{ Test } \\
\hline & & & $s_{\mathrm{MGM}}$ & $s_{\mathrm{WLS}}$ & $s_{\mathrm{M} 1}$ & $s_{\mathrm{M} 2}$ \\
\hline \multirow{4}{*}{$\begin{array}{l}\Omega_{1}: \gamma_{1}=1, \\
\gamma_{2}=1, \gamma_{3}=2\end{array}$} & Constant & 128 & 0.987 & 0.987 & 0.985 & 0.985 \\
\hline & \multirow{2}{*}{16 balanced blocks } & 128 & 0.992 & 0.992 & 0.951 & 0.951 \\
\hline & & 256 & 1.000 & 1.000 & 1.000 & 1.000 \\
\hline & HeaviSine & 300 & 1.000 & 1.000 & 1.000 & 1.000 \\
\hline $\begin{array}{l}\Omega_{2}: \gamma_{1}=1, \\
\gamma_{2}=3, \gamma_{3}=3\end{array}$ & Constant & 128 & 1.000 & 1.000 & 1.000 & 1.000 \\
\hline \multirow{2}{*}{$\begin{array}{l}\Omega_{3}: \gamma_{1}=4, \\
\gamma_{2}=3, \gamma_{3}=2 \\
\Omega_{4}: \gamma_{1}=1\end{array}$} & \multirow[t]{2}{*}{16 balanced blocks } & 128 & 1.000 & 1.000 & 1.000 & 1.000 \\
\hline & & 256 & 1.000 & 1.000 & 1.000 & 1.000 \\
\hline$\gamma_{2}=1, \gamma_{3}=4$ & HeaviSine & 300 & 1.000 & 1.000 & 1.000 & 1.000 \\
\hline
\end{tabular}

Table 1 that $\hat{\sigma}_{\mathrm{DJ}}^{2}$ performed poorly in estimating $\sigma^{2}$. Thus, among the four possible MGM, WLS, DJ and Müller approaches, the proposed two MGM and WLS approaches may be recommended for testing for the heteroscedasticity in wavelet regression models as these approaches appear to perform quite well in controlling the size as well as lead to highly satisfactory power levels.

The powers under the $t$-distribution were generally large for all functions although in reality the power would be smaller if the size is adjusted to the correct nominal level.

\section{Some remarks on an alternative test}

As the assumption of normality for the noises seem to be quite reasonable in practice, any nonparametric test for such cases will be less efficient (in the sense of size and power) than the normal based test such as the score test discussed in this paper. Nevertheless, we consider another normality based but simpler test, namely, Bartlett (1937) test for testing the equality of the variances. Note however that as we estimate the nonparametric function by wavelet based approaches, the sample variances required to compute the Bartlett test are now computed by using the values of the estimated function in place of sample means. We therefore refer to this test as the modified Bartlett test (MBT). For testing $H_{0}: \sigma_{(1)}^{2}=\cdots=\sigma_{(q)}^{2}$, this MBT statistic may be simplified as

$$
M=\frac{-2 \log l}{1+1 / 3(q-1)\left\{\sum_{j=1}^{q}\left(1 / n_{j}\right)-(1 / n)\right\}},
$$

where $-2 \log l=n \log S^{2}-\sum_{j=1}^{q} n_{j} \log S_{j}^{2}$ with $S_{j}^{2}=\sum_{k=1}^{n_{j}}\left(y_{j k}-\hat{f}_{j k}\right)^{2} / n_{j}$ and $S^{2}=\sum_{j=1}^{q} n_{j} S_{j}^{2} / \sum_{j=1}^{q} n_{j}$. 
For details on the derivation of the $M$ statistic in (5.1) we refer to Sutradhar (1996) for convenience. When sizes and powers of this test were compared to the normality based test, Bartlett's test was found to trail the score test in both size and power performances especially for the balanced block and HeavySine functions.

\section{Conclusion}

Results of this paper suggest that it is important to formulate the null hypothesis properly for testing for the heteroscedasticity in wavelet regression models. Also, it is crucial to estimate the constant wavelet variance under the null hypothesis consistently which then lead to a proper score test with satisfactory size and power performances.

\section{Acknowledgements}

This research was supported by grants from the Natural Sciences and Engineering Research Council of Canada. The authors wish to thank the referee for suggestions that improved the presentation of this paper.

\section{References}

Abraham, B., Ledolter, J., 1983. Statistical Methods for Forecasting. Wiley, New York.

Abramovich, F., Silverman, B.W., 1998. Wavelet decomposition approaches to statistical inverse problems. Biometrika 85, $115-129$.

Antoniadis, A., Gregoire, G., McKeague, I.W., 1994. Wavelet methods for curve estimation. J. Amer. Statist. Assoc. 89, $1340-1352$.

Bartlett, M.S., 1937. Properties of sufficiency and statistical tests. Proc. Roy. Soc. A 160, $268-282$.

Breusch, T.S., Pagan, A.R., 1979. A test for heteroscedasticity and random coefficient variation. Econometrica 47, 1287-1294.

Cai, Z., Hurvich, C.M., Tsai, C., 1998. Score tests for heteroscedasticity in wavelet regression. Biometrika 85, $229-234$.

Donoho, D.L., Johnstone, I.M., 1995. Adapting to unknown smoothness via wavelet shrinkage. J. Amer. Statist. Assoc. 90, 1200-1224.

Härdle, W., Kerkyacharian, G., Picard, D., Tsybakov, A., 1998. Wavelets, Approximation, and Statistical Applications. Springer-Verlag, New York.

Hart, J.D., Wehrly, T.E., 1986. Kernel regression estimation using repeated measurements data. J. Amer. Statist. Assoc. 81, 1080-1088.

Koenker, R., 1981. A note on studentizing a test for heteroscedasticity. J. Econometrics 17, $107-112$.

Kovac, A., Silverman, B.W., 2000. Extending the scope of wavelet regression methods by coefficient dependent thresholding. J. Amer. Statist. Assoc. 95, 172-182.

Moran, P.A.P., 1970. On asymptotically optimal tests of composite hypotheses. Biometrika 57, 47-55.

Müller, H.G., 1985. Empirical bandwith choice for nonparametric kernel regression by means of pilot estimators. Statist. Decisions 2, 193-206 (Supplementary Issue).

Neyman, J., 1959. Optimal Asymptotic Tests of Composite Hypotheses. Probability and Statistics: The Harald Cramer Volume. Ulf Grenander, Wiley, New York, pp. 213-234.

Oyet, A.J., Wiens, D.P., 2000. Robust designs for wavelet approximations of regression models. J. Nonparametric Statist. 12, 837-859. 
Silverman, B.W., 1985. Some aspects of the spline smoothing approach to nonparametric regression curve fitting. J. Roy. Statist. Soc. Ser. B 47, 1-52.

Sutradhar, B.C., 1996. Score test versus Bartlett type modified test for testing homogeneity of variances with autocorrelated errors. Sankhya B 58, 10-27.

Sutradhar, B.C., Dagum, E.B., 1998. Bartlett-type modified test for moving seasonality with applications. The Statistician 47, 191-206. 